\title{
SIMULAÇÃO DA EXPECTATIVA DE PERDAS DE SOLO EM MICROBACIA SOB DIFERENTES MANEJ OS FLORESTAIS ${ }^{(1)}$
}

\author{
A.G. CASTRO ${ }^{(2)} \&$ M. VALÉRIO FILHO ${ }^{(3)}$
}

\begin{abstract}
RESUMO
Este estudo foi designado a avaliar, por meio de simulação cartográfica em Sistema de Informações Geográficas (SIG), o impacto de diferentes estratégias de manejo florestal na escala de uma microbacia hidrográfica. 0 projeto foi desenvolvido em uma pequena microbacia $\left(2,8 \mathrm{~km}^{2}\right)$, localizada na porção norte do litoral capixaba (Estado do Espírito Santo), inserida em um a área de plantação industrial de eucalipto pertencente ao complexo agroindustrial da Aracruz Celulose S.A. A avaliação dos impactos potenciais foi efetuada por meio da aplicação da equação universal de perdas de solo (EUPS), sendo diferentes cenários simulados com base no processamento digital da base primária de dados (dados ancilares relativos a cobertura pedológica, relevo, clima e vegetação). A si mulação considerou quatro diferentes estratégias de manejo, variando de uma situação mais crítica (completa ausência de qualquer técnica conservacionista) até uma abordagem mais próxima da situação atualmente observada (onde diferentes técnicas de manejo têm sido empregadas). Os primeiros resultados permitem denotar a adequacidade do uso dessas técnicas de investigação prospectiva em suporte ao manejo operacional de florestas de produção. Todavia, al guns cuidados devem ser adotados quanto ao alcance desta abordagem, especificamente quanto ao modelo EUPS e, ainda, a uma tendência à dispersão multiplicativa de erros detectada durante o processamento digital.
\end{abstract}

Termos de indexação: erosão, equação universal de perdas de solo, sistema de informações geográficas, si mulação, floresta de produção.

\section{SUMMARY: SOI L LOSS EXPECTANCY IN A WATERSHED UNDER DIFFERENT FOREST MANAGEMENTS}

A cartographic simulation in a Geographical I nformation System (GIS) was used to evaluate the environmental impacts of different forest management strategies on a watershed scale The project was conducted in a small $\left(2 \mathrm{~km}^{2}\right)$ watershed located at northern coast of the State of E spírito Santo, Brazil, which is inserted in an area of eucalypt-clone plantation comprised by theagro-industrial complexAracruz CeluloseS.A. Potential impacts wereassessed by applying

\footnotetext{
(1) Recebido para publicação em março de 1996 e aprovado em maio de 1997.

(2) Ecólogo, Estudante de Doutorado em Ciências da Engenharia Ambiental, Centro de Recursos Hídricos e Ecologia Aplicada, EESC, Universidade de São Paulo, Caixa Postal 359, CEP 13560-590 São Carlos (SP).

(3) Engenheiro-Agrônomo, Pesquisador Titular, Instituto Nacional de Pesquisas Espaciais, Caixa Postal 515, CEP 12227-010 São J osé dos Campos (SP). Bolsista do CNPq.
} 


\begin{abstract}
the Universal Soil Loss Equation (USLE) in a GIS, where different scenarios were simulated by primary data processing (auxiliary data retating to the surface soil, relief, climate and vegetation). The simulation considered four different management strategies: from the worst possiblesituation, such as the completeabsence of any conservati on practice, to a more real istic assumption where management techniques are currently applied. First results demonstrated theadequacy of thesetechnol ogies for prospectiveinvesti gations and as guidel ines to operational management of forest plantations. Nevertheless, cauti on must betaken concerning the scope of this approach, specially regarding the USLE mode, and the multiplicative error dispersion trend during data processing.
\end{abstract}

Index terms: erosion, soil Ioss, USLE, GIS, simulation, modelling, forest.

\section{INTRODUÇÃO}

Atividades de exploração intensiva de recursos naturais, a exemplo de florestas de produção de madeira para indústrias de papel e celulose, são, correntemente, vistas como elementos de alto potencial de impacto ambiental e econômico (Chang et al., 1994; Larney et al., 1995). As formas de contornar tal tendência centram-se, de imediato, na adequação dos planos de manejo às condições previamente observadas no ambiente, em particular quanto ao grau de fragilidade ou suscetibilidade a determinadas classes de distúrbios.

Processos de erosão e perdas desol o são entendidos como um dos principais testemunhos de procedimentos e estratégias errôneas de manejo e produção (Guerrant et al., 1991; Prato \& Wu, 1991; Olson et al., 1994; Soileau et al., 1994). Além dos danos diretamente ocasionados pela perda do substrato edáfico sobre a produtividade de culturas, este processo é responsabilizado por inúmeros distúrbios difusos, em particular comprometimento de recursos hídricos e aceleração de processos mais amplos de degradação ambiental (Ashraf \& Borah, 1992; Mostaghini et al., 1994; Agnihotri \& Yadav, 1995) . A forma mais direta de controle da erosão trata da implementação de práticas conservacionistas, dentre as quais se destaca o manejo de unidades hidrográficas como a mais promissora. Todavia, para que tais ações resultem em melhora significativa a custos aceitáveis, é necessário que se proceda a um reconhecimento do contexto ambiental ondeestas devam ser implantadas e a uma caracterização das inter-relações entre as unidades funcionais componentes do espaço rural (Fu \& Gulink, 1994; Quine et al., 1994).

A adoção de técnicas de processamento digital, em particular processamento de dados e informações geográficas, atribui às investigações ambientais características e potencialidades realmente integradas, embora, algumas vezes, careçam de objetividade (Sharma \& Singh, 1995). A utilização de model os matemáticos - conceituais, determinísticos e estocásticos - apresenta-se como alternativa válida para otimização desses processos de integração, emprestando as iniciativas em SIG's objetividade e orientação mais direta aos problemas investigados.

I números exemplos podem ser encontrados quanto à utilização da equação universal de perdas de solo
(EUPS) em estudos prospectivos, provando ser este um dos instrumentos analíticos mais bem sucedidos para estudos de processos de erosão, em especial superficiais (Lo et al., 1988; McCool et al., 1989; Donzeli et al., 1992; Mellerowic et al., 1994). Sua utilização em conjunto com sistemas digitais é cada vez mais freqüente, embora ainda persistam entraves e lacunas, em especial quanto à adequacidade de utilização em diferentes escalas e contextos ambientais.

Quando comparado com outras classes de modelos, em especial determinísticos, esse modelo (EUPS) apresenta vantagens operacionais, em especial por não exigir um número el evado de parâmetros e permitir sua implementação em sistemas de informações geográficas com relativa facilidade (Pelletier, 1984; Savabi, 1993). Tem-se mostrado, ademais, ser extremamente útil em iniciativas voltadas ao zoneamento ambiental em escalas e procedimentos menos detalhados e, ainda, como instrumento de simulação. Ressalta-se, todavia, que deve ser utilizado considerando-se as características e limitações próprias da estrutura lógica e experimental, em especial tendo-se em vista os propósitos originais para os quais foi desenvolvido. Recomendações e uma avaliação de suas reais potencialidades podem ser encontradas em estudos desenvolvidos por seus autores, em especial em Wischmeier \& Smith (1978) eWischmeier (1976).

Este estudo foi designado a um primeiro zoneamento ambiental com base na estimativa do potencial natural e expectativa de perda de solos, obtidas por meio de simulação cartográfica em SIG. O objetivo a médio prazo trata do desenvolvimento de método em análise ambiental que permita: (1) inclusão de estudos de expectativa de erosão em procedimentos de manejo intensivo de florestas de produção; (2) estabelecimento de correlações entre técnicas de manejo e processos hidrológicos em escala de microbacia, e (3) análises de risco de impactos ambientais em suporte ao planejamento e operação do manejo florestal.

O delineamento experimental deste estudo foi definido de forma a permitir a simulação e análise comparativa da expectativa quanto aos processos de perda de solo por deflúvio superficial em diferentes condições de manejo ambiental eflorestal. O conjunto de resultados obtidos a partir dessas simulações, de 
refinamentos experimentais e observações de campo, torna possível o aprimoramento das ações de manejo florestal, em especial quanto ao estabel ecimento de práticas ótimas de manejo em programas de gestão integrada da base de recursos naturais, incluindo os florestais.

\section{MATE RIAL E MÉTODOS}

O local de estudo compreende uma microbacia inserida na bacia hidrográfica do Córrego Guaxindiba, município de Aracruz (ES), caracterizada por cobertura pedológica predominantemente podzólica, relevo suave a fortemente inclinado, com dissecamentos característicos de áreas de tabuleiros da região costeira norte do Estado do Espírito Santo. A cobertura vegetal é composta, basicamente, por florestas de produção em áreas de relevo suave a levemente ondulado (clones de eucalipto) e por formações resi duais defloresta nativa - Mata Atlântica - representadas por diferentes estádi os regenerativos, de capoeirão a capoeira.

O procedimento de integração de dados foi orientado segundo modelo equação universal de perdas de solo (Wischmeier \& Smith, 1978), compreendendo cinco etapas, a saber: (1) verificação e ajuste da base primária de dados; (2) obtenção de informações complementares a partir de um primeiro processamento digital dos dados primários; (3) cálculo do potencial natural de erosão; (4) integração desse com parâmetros antrópicos (CP) para avaliação das taxas de expectativa de perda de solo (Índice A), e (5) refinamento dos procedimentos de processamento e análise dos dados, em especial quanto à utilização da equação universal de perdas de solo em SIG. As duas primeiras etapas representaram a definição e organização de uma base primária de dados. $\mathrm{Na}$ terceira e quarta etapas, efetuaram-se procedimentos de modelagem e classificação vol tados ao zoneamento ambiental da microbacia, enquanto, na quinta, procurou-se verificar a adequacidade do método de simulação segundo objetivos específicos previamente estabelecidos.

Etapa 1 - Procedeu-se a uma verificação da base de dados original, representada pelo mapa de cobertura pedológica e mapeamento topográfico, ambos em escala média de 1:2.500. O projeto em SIG foi definido segundo escala média da base de dados original (1:2.500), adotando-sea projeçãoUTM (datum SAD 69). A partir de dados planialtimétricos, foi gerado um modelo numérico de terreno (MNT) por meio de manipulação analítica no SIG, sendo, concomitantemente, efetuados ajustes quanto ao dimensionamento da grade analítica e redução da redundância dos dados. Essemodelo foi gerado a partir de uma grade regular com resolução de $3 \times 3$ metros, posteriormente reamostrada para $5 \times 5$ metros. $O$ mapa de solos foi digitalizado em formato vetorial, sendo, a seguir, transposto para formato raster em resolução espacial idêntica à adotada para o MNT. Definiu-se, assim, a resolução espacial dos dados em SI G, adotada para as etapas de manipulação analítica e de apresentação final .

Etapa 2 - Nesta, foram gerados os mapas de declividade e comprimento de vertentes (fatores $\mathrm{S}$ e L da EUPS). I nicial mente, procedeu-seà manipulação do MNT, a partir da qual foram obtidos mapas de declividade e aspecto (orientação de vertentes), segundo equação proposta em Burrough (1986). Os planos de informação resultantes foram, a seguir, classificados segundo os interval os:

Declividade: 0-3\%, 3-6\%, 6-12\%, 12-20\%, $20-$ $40 \%, 40-60 \%,>60 \%$.

Aspecto: 0-45o, 45-90, 90-135o, 135-180, 180$225 \circ, 225-270 \circ, 270-315 \circ, 315-360$.

Com base em um mapa de drenagem, derivado de mapeamento topográfico em escala 1:2.500, foi gerado um Mapa de Distância da Rede de Drenagem caracterizando as faixas de eqüidistância a partir da linha de drenagem. O cruzamento desse com o de orientação de vertentes (aspecto) permitiu o cál culo do fator $L$ (comprimentos de vertentes), classificado segundo distância preponderante dentro de um grupo de vertentes nos seguintes intervalos: 0-50, 50-100, 100-200, 200-500 e > 500, em metros.

Na medida em que o mapeamento pedológico da microbacia apresentou predominância de solos podzólicos, os valores adotados para o fator $\mathrm{K}$ representaram uma média para solos desta classe, expresso como $K=0,0586$ t ha-1 R (Bertoni \& Lombardi Neto, 1992). À parametrização do fator $R$ corresponderam três valores de referência para ofator $\mathrm{R}$, concernentes a dezembro ejunho, respectivamente, maior e menor intensidade de chuvas para a região, e ainda um valor médio anual. Esses valores foram calculados segundo método proposto em Bertoni \& Lombardi Neto (1992) a partir de série histórica regional (média de vinte anos de observação).

Para a parametrização do fator proteção do solo conferida pela cobertura vegetal (C), considerou-se sua variação segundo tipo de cobertura (floresta de produção composta por cl ones de eucalipto e floresta nativa, representada por formações secundárias de Mata Atlântica), bem como o estádio de desenvolvimento das florestas de produção (plantio, cobertura com menos de um ano de idade e cobertura com mais de um ano). Os valores atribuídos à cobertura de florestas de produção corresponderam a $\mathrm{Cp}=0,90$ (fase de plantio - $0 \%$ recobrimento do solo), $\mathrm{Ci}=0,28$ (fase intermediária - $60 \%$ recobrimento do solo) e Cf $=0,05$ (fasefinal - 90\% recobrimento do solo). Para cobertura nativa, atribuiu-se um único valor, $C n=0,004$, não considerando variações estruturais a cobertura. Esses val ores foram, à semel hança do fator $\mathrm{K}$, obtidos a partir de relatório técnico de consultoria do Dr. Lombardi Neto à Aracruz Celulose. 
Os valores para o fato P (práticas conservacionistas) foram definidos com base em três situações distintas. A primeira expressa completa ausência de práticas conservacionistas ea utilização de técnicas convencionais de preparo do solo (aração e gradagem), assumindo valor de $P=1,0$. A segunda foi definida segundo variação dos valores locais de declividade e a utilização de técnicas de plantio em nível, correspondendo a $\mathrm{P}=0,50$ para declividades $<$ $3 \%$ até $P=1,0$ para declividades $>12 \%$. A terceira, mais próxima do observado, considerou, além do plantio em nível, a implementação de técnicas de plantio direto por coveamento. Foi definida por meio da multiplicação dos valores anteriores ( $P$ variando segundo declividade do terreno) pela constante CV $=0,20$ (Lombardi Neto, comunicado pessoal).

Etapa 3 - Com base nos dados gerados em etapas pregressas, calculou-se o potencial natural de erosão a partir dos parâmetros físicos da EUPS - erosividade $(\mathrm{R})$, erodibilidade (K), declividade (S) e comprimento de vertentes $(L)$, conforme equação:

$$
\mathrm{PNE}=\mathrm{R} * \mathrm{Ft} * \mathrm{~K}
$$

onde: $\mathrm{R}$ representa a erosividade ou potencial erosivo das chuvas; Ft, o fator topográfico resultante da integração dos parâmetros morfométricos (L eS) e K, a erodibilidade dos solos. $\mathrm{O}$ fator topográfico $(\mathrm{Ft})$ foi calculado segundo equação proposta em Bertoni \& Lombardi N eto (1992):

$$
\mathrm{Ft}=\mathrm{S} 1,18 * \mathrm{~L} 0,63 * 0,00984
$$

onde: S representa valores médios de declividade (\%) e $L$, intervalos de comprimento das vertentes (em metros).

Com base nos valores máximo, médio e mínimo de erosividade (R Máx, R Méd e R Mín), obtiveram-se os valores correspondentes de potencial natural de erosão (PNE's), expressos como: PNE Máx (segundo R Máx $=1246$ MJ mm ha-1 h-1 ano-1), PNE Méd

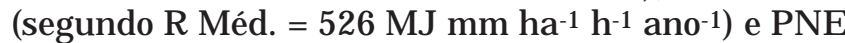
Mín (segundo R Mín = 110 MJ mm ha-1 h-1 ano-1). Os valores de erosividade (R), obtidos a partir de relatório de consultoria técnica do Dr. Lombardi Netoà Aracruz Celulose, foram calculados conforme método exposto em Bertoni \& Lombardi N eto (1992) (Lombardi Neto, comunicado pessoal).

Etapa 4 - Nesta, procedeu-se à simulação de diferentes cenários, calculando-se os valores de expectativa de perda de solo (Índice A) de acordo com variações dos fatores $R, C$ e $P$ da EUPS. O primeiro cenário (i) consistiu no cálculo da expectativa de perda de solos com base nos valores máximos de potencial natural (PNE Máx.), considerando-se, ainda, completa ausência detécnicas conservacionistas $(P=1,0)$ e três diferentes valores para o parâmetro C. Estes corresponderam ao percentual de recobrimento do solo reconhecidos para as fases de plantio $(P)(0 \%$ de recobrimento do solo), fase intermediária (I) $(60 \%$ de recobrimento do solo) e fase final (F) (90\% de recobrimento do solo). Neste cenário, procedeu-se, em última instância, apenas à variação dos valores correspondentes ao fator $\mathrm{C}$, atribuindo-se um único valor para os demais parâmetros da EUPS. Objetivouse, assim, verificar o impacto ocasi onado por diferentes percentuais de recobrimento do solo sobre os índices de perda de solo projetada pelo modelo.

O segundo cenário (ii) diferiu do anterior somente quanto aos valores atribuídos ao fator P. Este sofreu variações referentes ao padrão de declividade do terrenoe, ainda, por consi derar a utilização detécnicas de plantio em nível. À semelhança do cenário (i), adotaram-se valor máximo de PNE (considerando R máximo) etrês valores para ofator $C$, correspondentes aos diferentes percentuais de recobrimento do solo (função do estádio de desenvol vimento da floresta de produção).

No terceiro cenário (iii), procurou-se avaliar a influência só do fator erosividade sobre o índice de expectativa de perda de solos, sendo, para tal, adotado um valor médio (PNE Méd), mantendo-se os valores de $P$ atribuídos ao cenário 2 e definindo-se apenas um valor para o fator $\mathrm{C}$, correspondente à condição de recobrimento observada na fase de plantio.

No quarto cenário (iv), consideraram-se PNE Máx e valores do fator $\mathrm{P}$ correspondentes ao sistema de plantio por coveamento, incluindo-se, ainda, valor específico para o fator $\mathrm{C}$ correspondente à cobertura floresta nativa.

Etapa 5 - Nesta, foram extraídas informações quantitativas dos mapas temáticos derivados do processo de mani pulação analítica em SI G, efetuandosea anál ise de correlação cruzada entreal guns planos de informação, planimetria das classes de expectativa de perda de solos para os diferentes cenários de simulaçãoe, ainda, análises estatísticas paramétricas e exploratórias da base final de informações.

\section{RESULTADOS E DISCUSSÃO}

Os resultados desta simulação são considerados como elementos prospectivos, podendo ser utilizados em um primeiro zoneamento ambiental com base na similaridade dos valores encontrados para oíndice de expectativa de perda nos diferentes cenários de simulação. A variação planimétrica de cada uma das classes indica, de forma indireta, o efeito esperado em função da mudança dos valores deal guns parâmetros. Apenas aquel es classificados como antrópicos (C e P) e o fator erosividade (R) foram considerados para simulação, permanecendo constantes os demais.

A figura la indica que a participação percentual (sobre a área da microbacia) das classe de PNE sofre uma influência significativa da erosividade, não obstante as diferenças observadas entre os valores de erosividade $\mathrm{R}$ máximo, médio e mínimo tenham sido superiores a 100\%. O potencial mínimo (PNE Mín) indica maior participação relativa de valores considerados baixos (classe 1), cerca de 97\% da área da mi crobacia. O índice PNE médio apresenta maior dispersão, passando a classe 1 a representar cerca de $58 \%$, com uma freqüência significativa de áreas consideradas como de alto potencial - cerca de $9 \%$. 
Para a situação mais crítica, valores mais altos de erosividade (R Máx.), observou-se uma redução significativa na participação das classes de potencial fraco a moderado, em contrapartida a uma maior freqüência das classes de forte a muito forte potencial natural (classes 4 a 6), as quais passam a perfazer cerca de $45 \%$ da área. Esses resultados indicam uma participação relativa importante do fator erosividade nos índices de suscetibilidade na medida em que os demais parâmetros foram mantidos constantes.

A figura $1 b$ representa a influência exercida por diferentes técnicas de manejo sobre o índice de expectativa de perda de solo (A). As variações observadas deveram-se tão-somente à utilização de diferentes estratégias ou práticas conservacionistas, descritas como: AP1 - completa ausência de práticas de conservação de solos; AP2 - plantio em nível, e AP4 - plantio em nível e por coveamento, equivalente ao plantio direto no manejo florestal. Nas três situações, foram considerados a condição de máxima erosividade e os valores de $C$ relativos à fase de plantio - praticamente sem recobrimento do solo. Variação mais expressiva foi observada entre o conjunto AP1/ AP2 e AP4, com maior participação das classes de expectativa de perda de solo fraca a moderada. Embora diferindo significativamente $(P<0,05)$, os cenários AP1 e AP 2 apresentaram tendência semelhante quanto à freqüência de índices intermediários a altos de expectativa de perda de solo. A microbacia foi, em sua quasetotal idade, classificada como área crítica, quanto à expectativa de perdas, tomando-se como base um valor tolerável de perdas em torno de 50 t ha-1 ano-1.

Para o cenário AP4, apenas cerca de $20 \%$ da microbacia foi classificada como potencialmente crítica, geralmente em áreas de maior declividade. Esses resultados reforçam a idéia de que, mesmo em situações de maior severidade (erosividade máxima), a introdução de técni cas conservacionistas apresentase como estratégia eficiente para redução da expectativa ou suscetibilidade potencial de perda de solo.

Simulando-se a variação do fator $\mathrm{C}$ em condição de máxima erosividade e ausência de práticas conservacionistas (Figura 2a), observou-se sua influência relativa sobre o índice $A$. E ntre as fases de plantio (AP1) e de desenvolvimento intermediário (AI 1), projetou-se uma redução decerca de $25 \%$ quanto à planimetria das áreas mais críticas, registrando-se uma retração significativa das áreas classificadas como de expectativa de perda de sol o alta a muito alta (classes 6 a 8). Na fase de recobrimento final (AF 1), cerca de $65 \%$ da microbacia passou a ser classificada como área não crítica, apresentando expectativas de perda abaixo do limite tecnicamente aceitável (tolerável).

Conforme figura $2 a$, a situação projetada quando a vegetação atinge cerca de $60 \%$ de recobrimento do solo (AI 1) implicou inversão de posição entreas dasses 2, 3 e 5. A adoção da técnica de plantio em nível passa a ser notada de forma mais evidente nessa fase, quando ocorre um aumento significativo do valor absoluto atribuído à classe 2 e redução das classes 3 e 5. Com cerca de $90 \%$ de recobrimento (AF 1), observouse um aumento na participação relativa das classes de 1 e 2, em especial da primeira, que acusou um acréscimo em área superior a 400\%, concomitante a uma redução nas áreas correspondentes às classes 3 e 4. Esta última apresentou menor variação, mesmo considerando-se as mudanças projetadas para a cobertura do solo. Relação semelhante pode ser verificada na figura $2 \mathrm{~b}$, onde é apresentada a relação entretaxa de recobrimento eíndices de suscetibilidade sob condições de plantio em nível.

A figura 3 permite observar a influência conjunta dos fatores $\mathrm{C}$ eP sobre a expectativa de perda de solo, detectando-se três grupos principais. A maior similaridade dos cenários AP 1 - AP $2\left(r^{2}=0,73 \mathrm{P}<0,05\right)$ parece indicar menor participação das técnicas conservacionistas em condições de recobrimento reduzido. É inter essante observar, todavia, a formação de um grupo entre as condições representadas por plantio convencional sobre erosividade média (AP3) e a situação atribuída à taxa de recobrimento final 90\% (AF 2) com plantio em nível e, em menor instância, com plantio sem práticas conservacionistas (AF 1). A maior erosividade atribuída à condição AF 1 parece ser, em parte, anulada pelo maior recobrimento, resultando em índices de expectativa de perda desolo mais reduzidos.

Por fim, nota-se um distanciamento do conjunto de cenários AP 4 - AI $2\left(r^{2}=0,86 P<0,05\right)$ em relação aos demais, indicando, primeiro, que a introdução de práticas conservacionistas na microbacia pode representar uma estratégia eficiente, embora seu padrão se assemelhe ao alcançado em estádio intermediário de recobrimento sob plantio em nível. Este pode ser um indicativo de que as condições de maior severidade, em especial nos Iocais com declividade $>12 \%$, apresentam-se como fatores restritivos ao uso, independentemente, do emprego de técnicas conservacionistas. A formação de um terceiro conjunto, representado pelos cenários AP3 AF 2 - AF 1, pode indicar que o fator erosividade, à semelhança do topográfico, constitui importante função de força quanto à projeção dos índices de expectativa de perda de solo. Nota-se, todavia, que desses apenas a correlação entre AP3 e AF 1 foi reconhecida como significativa ( $\left.r^{2}=0,84 ; P<0,05\right)$.

A correlação cruzada representa outra forma de análise dos resultados, em especial quanto à interrelação espacial entre os parâmetros da EUPS e índices resultantes. A distribuição das classes de aspecto ou orientação de vertentes, p.e., não obedeceu a um padrão definido quanto à declividade, ocorrendo uma distribui ção eqüitativa entreas diversas dasses. Quanto ao comprimento de rampa, observou-se maior concentração das classes 3 e 4 emáreas de decli vidade reduzida $(0-3 \%)$, relevo mais movimentado relacionado com menores comprimentos de rampa.

O índice PNE Máx. revela maior freqüência (em área relativa) das dasses de declividade 1, 2 e, em 


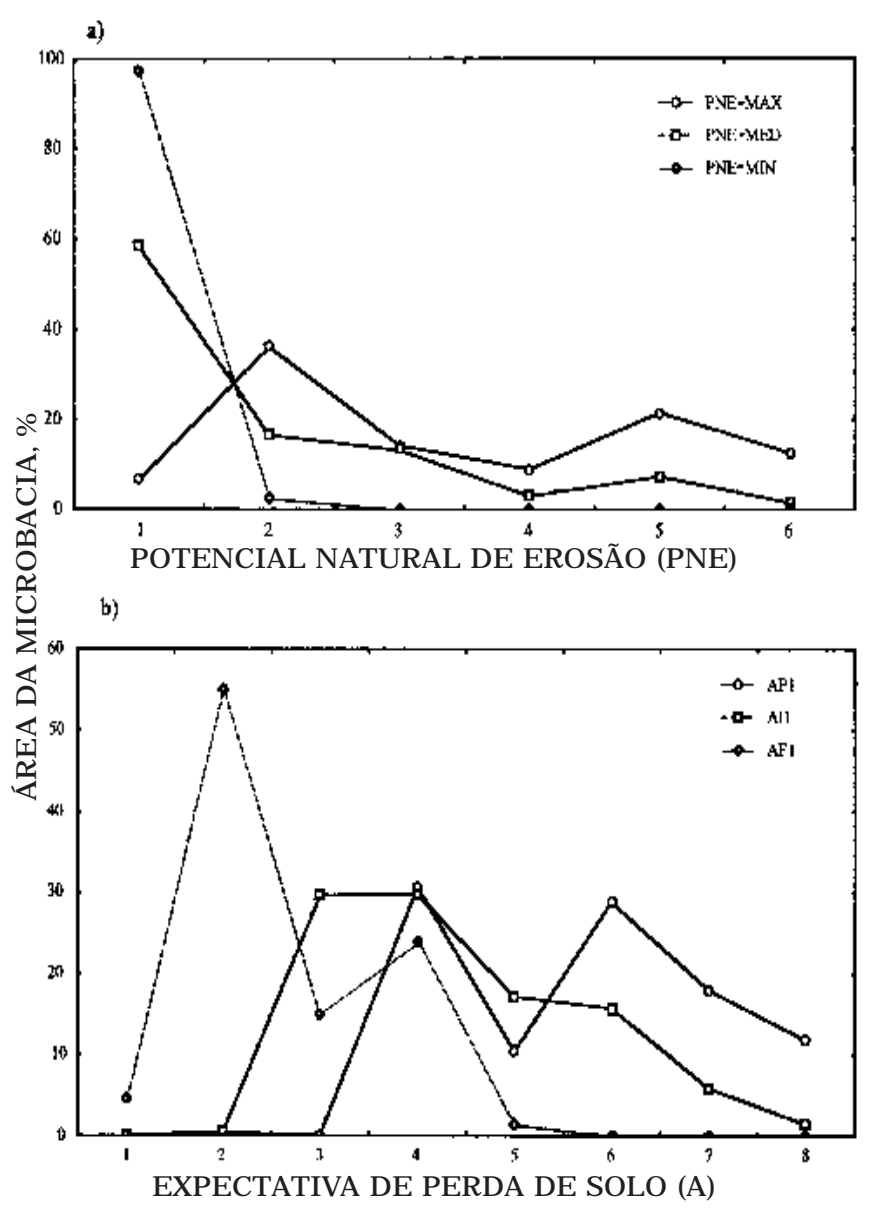

Figura 1. Distribuição relativa das classes de PNE segundo variação dos valores de erosividade (R) expressos de acordo com percentual sobre área da microbacia.

menor escala, sobrea classe 6, indicando, ainda, maior concentração de índices baixos a moderados de expectativa de perda de sol o em áreas com declividade mais reduzida (classes 1 e 2). 0 índice PNE médio apresentou uma freqüência mais acentuada da dasse 1 , baixo potencial, em áreas de relevo suave a suaveondulado (classes declividade 1 e 2), apesar de ocorrer uma zona de sobreposição de cerca de $0,20 \mathrm{~km}^{2} \mathrm{em}$ áreas com declividade média, entre 6 e $12 \%$. Observouse, ainda, uma sobreposição da classe de expectativa 3 (cerca de 0,31 km²) em áreas com relevo mais movimentado, e cerca de $0,16 \mathrm{~km}^{2}$ da classe 5 , em condições idênticas de relevo.

A correlação cruzada deAP 1 e declividade indicou maior concentração em áreas de menor declividade, embora sejam observadas parcelas significativas em áreas de maior declive. Todavia, o registro de expectativa de perda de solo alta em áreas de relevo suave a suave-ondulado pode indicar uma participação efetiva do fator comprimento devertentes na defini ção final dos índices de expectativa de perda de solo. Essa tendência permanece em relação às outras duas componentes da série (AI 1 eAF 1 ), embora ocorra um deslocamento no sentido de expectativas mais reduzidas de tais perdas. A correlação das fases de plantio e intermediário com padrões de declividade indica uma relação constante entre as classes de expectativa mais el evadas ezonas de declividade mais acentuada. Tal tendência modifica-se somente quanto a AF 1, fase final de desenvol vimento da cobertura florestal, quando ocorre um deslocamento do índice de expectativa para áreas menos movimentadas. Para AP4, observou-se uma concentração nas classes de declividade 1, 2 e 6, representando, esta última, condições de rel evo bastante movimentado. Este fato sugere que, mesmo em áreas mais movimentadas, a expectativa de perda é reduzida (classe 1 ) em função do emprego de técnicas conservacionistas mais eficientes, como no plantio direto por coveamento.

Correlacionando-se os resultados de diferentes séries de simulação, p.e., AP 1, AP2 e AP4, observa-se que ocorre variação espacial das classes de suscetibilidade, ademais daquelas esperadas pela simples redução das expectativas de perda de solos. Tal fato denota certa inconsistência lógica na caracterização dos limites entre as classes, podendo ser parcialmente atribuído ao formato de manipulação - grade regular e, ainda, a própria lógica do modelo utilizado.

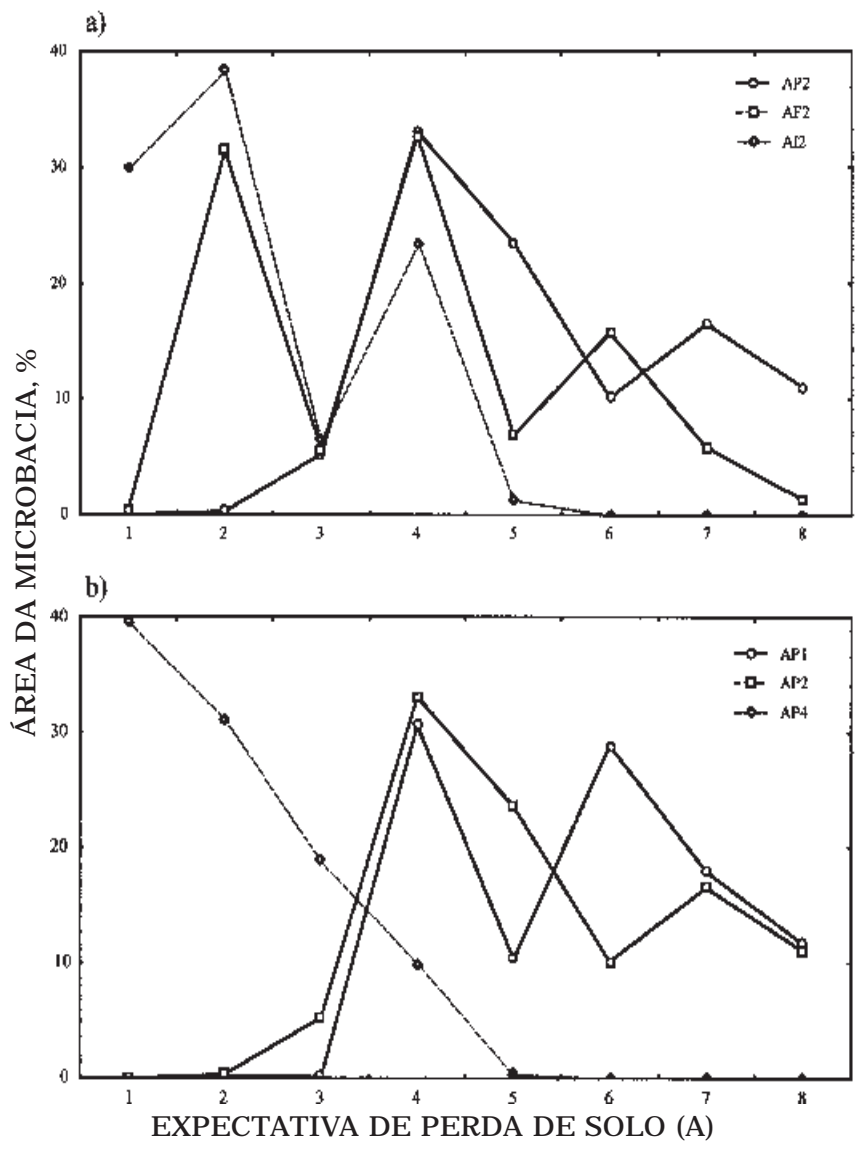

Figura 2. Participação relativa das classes de expectativa de perda de solo segundo variação dos valores do fator C (cobertura e manejo), sob condições extremas de potencial natural de erosão. 
Na obtenção de atributos primários da base de dados, situação mais crítica foi observada quanto aos dados de comprimento de rampa e declividade derivados do model o numérico de terreno (MNT), em especial por sua importância relativa nos resultados finais. E mbora se tenha realizado um primeiro ensaio quanto às melhores alternativas de confecção automática - via SIG - cabe maior detal hamento do método empregado. $\mathrm{O}$ ajuste deste apresenta-se estreitamente relacionado com as condições específicas do local de estudo, bem como da própria estratégia experimental adotada, incluindo-se, nesta, nível de detal he, desvio aceitável e escala detrabal ho.

A utilização de sistema SIG em procedimentos analíticos constitui método promissor na medida em que sejam estabelecidos alguns procedimentos de acompanhamento e controle de qualidade durante processamento e integração dos dados. A verificação da consistência e confiabilidade da base primária de dados, ademais, é de extrema importância, visto constituir a etapa mais dispendiosa ecrítica detodo o processo. Especialmente em investigações desse tipo, no qual se utilizaram matrizes com cerca de 198.000 elementos, a base de dados deve ser constantemente aferida quando à acuracidade e consistência confiabilidade e coerência dos dados obtidos nos diversos níveis de integração.

Deve-se atentar, ainda, para a questão da adequacidade do modelo adotado aos objetivos estabel ecidos. A aplicação do modelo EUPS forneceu uma base conceitual para o zoneamento preliminar, constituindo instrumento analítico para simulação de processos de perda de solo. Sua adequacidade, todavia, é reconhecida por meio das possibilidades de internalização das informações em programas de planejamento e manejo florestal. Apesar da ampla utilização deste model o nos mais diversos contextos e escalas, a adequacidade de sua aplicação permanece como elemento central de discussão. É necessário, todavia, conferir especial atenção à escala de generalização.

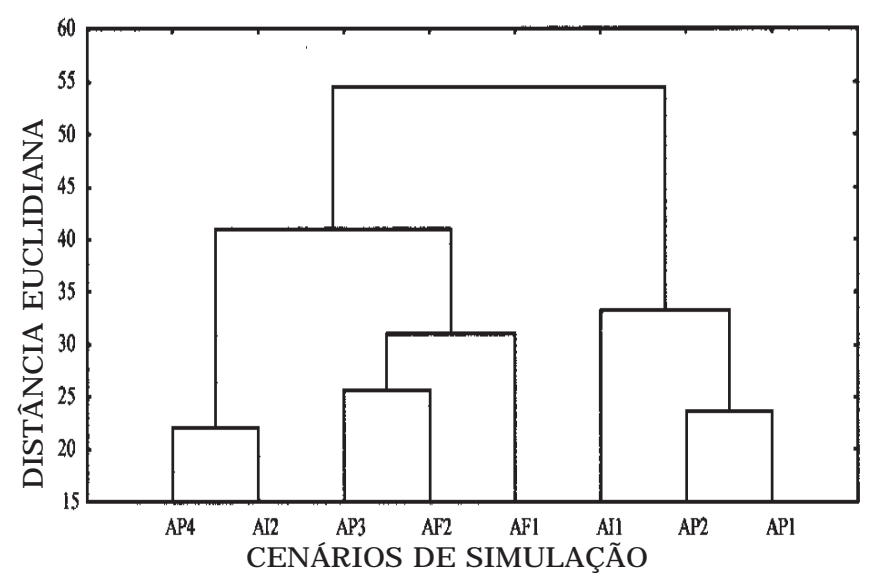

Figura 3. Análise de agrupamentos - distância euclidiana - considerando os diversos cenários de simulação.
A partir de uma tipologia estrutural do ambiente, foi possível simular diferentes contextos e proferir prognósticos quantoao impacto potencial de diferentes estratégias de manejo sobre a estabilidade do ambiente analisado. As inúmeras possibilidades de integração permitem, por exemplo, a avaliação e análise de riscos de contami nação e desenvol vimento conceitual/operacional de práticas ótimas de manejo ou unidades básicas de manejo.

\section{CONCLUSÕES}

Base de Dados - Dois aspectos merecem consideração: (1) nível de detal hamento dos dados e (2) compatibilização entre escala do projeto em SIG e nível de agregação dos dados e informações.

Método de Integração em SIG - O model o utilizado, EUPS, apresenta como vantagens a facilidade de implementação e interpretação dos resultados, embora a aplicação em SIG mer eça atenção especial, em particular quanto à dispersão de erros. A efetividade ou propriedade das análises realizadas e resultados obti dos é uma função direta da forma deinserção deste estudo em programas operacionais.

Modelo EUPS - Considerando-se a aplicação deste modelo em programas de manejo florestal, dois aspectos são de extrema rel evância: (1) os resultados al cançados representam uma aval iação semi quantitativa de caráter exploratório, e (2) deve-se atentar em especial para a dispersão multiplicativa de erros aleatórios e/ou sistemáticos advindos da própria estrutura do modelo e de sua aplicação em sistemas digitais de processamento de informações geográficas (SIG's).

Como elemento de investigação prospectiva, a aplicação da EUPS mostrou-se procedente, embora permaneça a questão de este model o estar restrito a uma avaliação semiquantitativa dos processos de perda de solos e apresentar restrições/limitações intrínsecas a sua estrutura (Wischmeier, 1976).

A abordagem recomendada constitui um aprofundamento dos procedimentos adotados neste estudo, para posterior derivação dos dados a partir de model os analíticos de estrutura matemática mais robusta. A aplicação desses modelos, todavia, requer um refinamento da base de dados e, sobretudo, o detal hamento de um plano experimental que permita: (1) validação dos resultados e (2) inserção efetiva das informaçõ̃es derivadas no âmbito do programa de manejo.

\section{AGRADE CIMENTOS}

Agradecemos à Empresa Aracruz Celulose S.A. o apoi o e suporte, sem os quais a realização deste estudo não seria possível. Em particular, às Gerências de Pesquisa, Floresta e M ei oAmbiente, cujos integrantes colaboraram decisivamente na el aboração e execução desta pesquisa. 


\section{LITERATURA CITADA}

AGNIHOTRI, R.C. \& YADAV R.C. Effects of different land uses on infiltration in ustifluvent soil susceptible to gully erosion. Hydrol. Sci. J ., 40:395-406, 1995.

ASHRAF, M.S. \& BORAH, D.K. Modeling pollutant transport in runoff and sediment. Trans. ASAE, St. J oseph, 35:1789-1797, 1992.

BERTONI, J . \& LOMBARDI NETO, F. Conservação do solo. 3.ed. São Paulo, Í cone, 1992. 395p.

BURROUGH, P.A. Principles of geographic information systems for land resources assessment. Oxford, Clareton, Press, 1986. $245 p$.

CHANG, C.C.; ATWOOD, J .D.; ALT, K. \& MCCARL, B.A. Economic impacts of erosion management measures in coastal drainage basins. J. Soil Wat. Conserv., Ankeny, 49:606-611, 1994.

DONZELI, P.L.; VALERIO FILHO, M.; PINTO, S.A.F.; NOGUEIRA, F.P.; ROTTA, C.L. \& LOMBARDI NETO, F. Técnicas de sensoriamento remoto aplicadas ao diagnóstico básico para planejamento e monitoramento de microbacias hidrográficas. Instituto Agronômico de Campinas, 1992. p.91119. (Documentos IAC, 29)

FU, B. \& GULINCK, H. Land evaluation in an area of severe erosion: Loess Plateau of China. Land Degrad. Rehab., Amsterdam, 5:33-40, 1994.

GUERRANT, D.G.; MILLER, W.W.; MAHANNAH，C.N. \& NARAYANAN, R. Soil processes and chemical transport. J . Environ. Qual., 20:396-402, 1991.

LARNEY, F.J .; IZAURRALDE, R.C.; ANZEN, H.H.; OLSON, B.M.; SOLBERG, E.D.; LINDWALL, C.W. \& NYBORG, M. Soil erosion - crop productivity relationships for six Alberta soils. J. Soil Wat. Conserv., Ankeny, 50:87-90, 1995.

LO, A.; EL-SWAIFY, S.A. \& ROSE, C.W. Analysis of erodibility of two soils using a process model. Soil Sci. Soc. Am. J ., Madison, 52:781-784, 1988

MCCOOL, D.K.; FOSTER, G.R.; MUTCHLER, C.K. \& MEYER, L.D. Revised slope length factor for the Universal Soil Loss Equation. Trans. ASAE, St. J oseph, 32:1571-1576, 1989.
MELLEROWICZ, K.T.; REES, H.W.; CHOW, T.L. \& GHANEM, I. Soil conservation planning at the watershed level using the Universal Soil Loss Equation with GIS and microcomputer technologies: a case study. J. Soil Wat. Conserv., Ankeny, 49:194-200, 1994.

MOSTAGHINI, S.; GIDLEY,T.M.; DILLAHA,T.A. \& COOKE, R.A. Effectiveness of different approaches for controlling sediment and nutrient losses from eroded land. J. Soil Wat. Conserv., Ankeny, 49:615-620, 1994.

OLSON, K.R.; LAI, R. \& NORTON, L.D. Evaluation of methods to study soil erosion-productivity relationships. J. Soil Wat. Conserv., Ankeny, 49:586-590, 1994.

PELLETIER, R.E. Evaluating nonpoint pollution using remotely sensed data in soil erosion models. J. Soil Wat. Conserv., Ankeny, 40:332-335, 1984.

PRATO, T. \& WU, S. Erosion, sediment, and economic effects of conservation compliance in an agricultural watershed. J. Soil Wat. Conserv., Ankeny, 46:211-214, 1991.

QUINE, T.A.; NAVAS, A.; WALLING, D.E. \& MACHIN, J. Soil erosion and redistribution on cultivated and uncultivated land near Las Bardemas in the Central Ebro River Basin, Spain. Land Degrad. Rehab., Amsterdam, 5:41-55, 1994.

SAVABI, M.R. Modeling subsurface drainage and surface runoff with WEPP. J . Irrig. Drain. Eng., New York, 119:801-813, 1993.

SHARMA, K.D. \& SINGH, S. Satellite remote sensing for soil erosion modeling using the ANSWERS model. Hydrol. Sci. J., Minnesota, 40:259-272, 1995.

SOILEAU, J.M.; TOUCHTON, J .T.; HAJ EK, B.F. \& YOO, K.H. Sediment, nitrogen, and phosphorus runoff with conventional - and conservation - tillage cotton in a small watershed. J. Soil Wat. Conserv., Ankeny, 49:82-89, 1994.

WISCHMEIER, W.H. Use and misuse of the universal soil loss equation. J. Soil Wat. Conserv., Ankeny, 31:5-9, 1976.

WISCHMEIER, W.H. \& SMITH, D.D. Predicting rainfall erosion losses - a guide to conservation planning. USDA/ARS, Washington, 1978. 58p. (Agricultural Handbook, 537) 